\title{
Diagnosis of Lumbar Foraminal Stenosis using Diffusion Tensor Imaging
}

\author{
Yawara Eguchi ${ }^{1}$, Seiji Ohtori ${ }^{2}$, Munetaka Suzuki ${ }^{1}$, Yasuhiro Oikawa ${ }^{3}$, Hajime Yamanaka ${ }^{1}$, \\ Hiroshi Tamai ${ }^{1}$, Tatsuya Kobayashi ${ }^{1}$, Sumihisa Orita ${ }^{2}$, Kazuyo Yamauchi ${ }^{2}$, Miyako Suzuki ${ }^{2}$, \\ Yasuchika Aoki ${ }^{4}$, Atsuya Watanabe ${ }^{4}$, Hirohito Kanamoto ${ }^{2}$, Kazuhisa Takahashi ${ }^{2}$ \\ ${ }^{1}$ Department of Orthopedic Surgery, National Hospital Organization Shimoshizu National Hospital, Yotsukaido, Japan \\ ${ }^{2}$ Department of Orthopedic Surgery, Graduate School of Medicine, Chiba University, Chiba, Japan \\ ${ }^{3}$ Department of Orthopaedic Surgery, Teikyo University Chiba Medical Center, Ichihara, Japan \\ ${ }^{4}$ Department of Orthopaedic Surgery, Eastern Chiba Medical Center, Togane, Japan
}

Diagnosis of lumbar foraminal stenosis remains difficult. Here, we report on a case in which bilateral lumbar foraminal stenosis was difficult to diagnose, and in which diffusion tensor imaging (DTI) was useful. The patient was a 52-year-old woman with low back pain and pain in both legs that was dominant on the right. Right lumbosacral nerve compression due to a massive uterine myoma was apparent, but the leg pain continued after a myomectomy was performed. No abnormalities were observed during nerve conduction studies. Computed tomography and magnetic resonance imaging indicated bilateral L5 lumbar foraminal stenosis. DTI imaging was done. The extraforaminal values were decreased and tractography was interrupted in the foraminal region. Bilateral L5 vertebral foraminal stenosis was treated by transforaminal lumbar interbody fusion and the pain in both legs disappeared. The case indicates the value of DTI for diagnosing vertebral foraminal stenosis.

Keywords: Diffusion tensor imaging; Familial lumbar stenosis; Fractional anisotropy

\section{Introduction}

Lumbar foraminal stenosis is a pathological condition in which degenerative changes of the vertebral column cause foraminal and extra foraminal entrapment of nerve roots and lumbar nerves. The dorsal root ganglion, which contains pain receptors, is also present at this site, causing marked pain, and the condition is intractable [1]. However, there is a hidden zone in this region [2], which remains easy to overlook even with advanced diagnostic imaging methods, and which is a factor in worsening the surgical outcome. Currently, the false-positive rate using magnetic resonance imaging (MRI) is approximately $30 \%$ to $40 \%$ when diagnosing lateral lesions of lumbar nerve roots that branch off from the spinal cord. This rate is extremely high and diagnosis is difficult [3]. A new method of diagnostic imaging is desired.

Diffusion weighted imaging (DWI) uses MRI to focus on the diffusion of water molecules. A motion problem gradient (MPG) is extracted and created by comparison to the diffusion movement of protons [4-7]. DWI is indispensable for the diagnosis of an acute cerebral infarction [8], and is currently widely-used in clinical practice $[9,10]$. DWI is strongly affected by the ease of diffusion of

Received Apr 7, 2015; Revised Apr 20, 2015; Accepted Apr 20, 2015

Corresponding author: Yawara Eguchi

Department of Orthopaedic Surgery, Shimoshizu National Hospital,

934-5, Shikawatashi, Yotsukaido, Chiba 284-0003, Japan

Tel: +81-43-422-2511, Fax: +81-43-421-3007, E-mail: yawara_eguchi@yahoo.co.jp 
water molecules and by the direction of the diffusion. Because the axonal cell membrane and myelin sheath in the nerve fibers prevent diffusion in the direction parallel to the nerve fiber fascicles, isotropy of the diffusion of water molecules is lost. This state is known as diffusion anisotropy, and selectively recording this information is known as diffusion tensor imaging (DTI) and tractography. The diffusion data can be used for the determination of a scalar fractional anisotropy (FA) value that reflects the directionality of the molecular diffusion. FA values range from 0 to 1 , with high $\mathrm{FA}$ values indicating anisotropic diffusion and low FA values indicating more isotropic diffusion. There is high diffusion anisotropy in interstitial medullated nerves as the direction of water molecule diffusion is limited to parallel to the nerve fibers due to the presence of a myelin sheath. However, diffusion anisotropy is reduced by demyelinating and degenerative conditions accompanying nerve injury, such as spinal cord injuries, and the FA value decreases. In recent years, the usefulness of DTI for demyelinating and degenerative diseases, such as multiple sclerosis, and chronic compressive lesions of peripheral nerves, such as carpal tunnel syndrome, has been reported [11-14]. We have reported that tract interruption occurs at the area of stenosis in patients with lumbar foraminal stenosis when investigated using DTI, with decreasing FA values [15]. Here, we report on a case of L5 foraminal stenosis that was difficult to diagnose and where DTI was useful for making the diagnosis.

\section{Case Report}

The patient was a 52-year-old woman. She had a chief complaint of low back pain, pain in the legs, predominantly on the right, numbness in bilateral foot soles and intermittent claudication after 20 minutes. The foot sole numbness, leg pain and low back pain started 5 years prior. She was first examined 4 years ago. Foraminal stenosis of L5 vertebrae was observed bilaterally; an L5 nerve root block was temporarily effective and the leg pain recurred. The patient was resistant to oral medications (tramadol hydrochloride, pregabalin). The nerve findings elicited using the manual muscle test were as follows (right side/left side): iliotibialis $5 / 5$, quadriceps $5 / 5$, tibialis anterior $4 / 5$, extensor hallux longus $4 / 5$, flexor hallus longus 5-/5-, gastrocnemius 5-/5. There was no reduction in sensation. The full term for the Japanese Orthopedic Association (JOA) score was 19/29 (normal score, 29 points); the visual analogue scale (VAS; from 100 [extreme amount of pain] to 0 [no pain]) for low back pain was 70; the VAS score for leg pain was 80 on the right and 50 on the left; and the VAS for leg numbness was 90 on the right and 50 on the left.

The patient had a history of a massive uterine myoma.

Bilateral L5 foraminal stenosis was observed on MRI and a massive uterine myoma was observed. Computed tomography (CT) images after L5 neuroscopy on the right showed a uterine myoma pressing on the sacroiliac joint from the front (Fig. 1). The central region of the spinal canal was normal with no compression on spinal myelogram CT. Bilateral L5 foraminal stenosis was observed, and an osteophyte was observed projecting into the foramen from the right $\mathrm{S} 1$ superior articular process, with the right L5 nerve root compressed (Fig. 2).

No abnormalities of either amplitude or nerve conduction velocity were observed in the tibial or peroneal nerves during electrophysiological studies (Fig. 3.)

We considered the sciatic neuralgia to be caused by the massive uterine myoma. One year after the initial examination, we performed a myomectomy. Leg pain was unchanged postoperatively.

To examine for L5 nerve injury due to foraminal stenosis in detail, we performed DTI using a Philips Achieva 1.5T MRI machine.

The DTI series were acquired using spectral presaturation with inversion recovery, and an echo-planar imaging sequence with a free-breathing scanning technique. Patients were scanned in a supine position using a SENSESpine-coil. The following imaging parameters were set: $800 \mathrm{sec} / \mathrm{mm}^{2}$ b-value, MPG: 15 directions, 10,000/71 ms for repetition time/echo time respectively, axial slice orientation, $3 / 0 \mathrm{~mm}$ slice thickness/gap, $320 \times 213 \mathrm{~mm}$ field of view (FOV), $96 \times 192$ matrix, $3.3 \times 1.66 \times 3.0 \mathrm{~mm}^{3}$ actual voxel size, $1.6 \times 1.6 \times 4.0 \mathrm{~mm}^{3}$ calculated voxel size, four excitations, 50 total slices, 10 minutes 31 seconds scan time. Regions of interest included the inside of the vertebral canal, nerve roots, and outside the foramen, and measured the nerve FA values on the affected and unaffected sides.

Interruption of tractography was observed at the L5 vertebral foramen on the right (Fig. 4). FA values (inside of the vertebral canal, nerve roots, and outside the foramen) were $0.434,0.459$, and 0.349 , respectively, on the right and $0.194,0.354$, and 0.472 , respectively, on the left.

Mean values in five healthy individuals were $0.436 \pm$ 

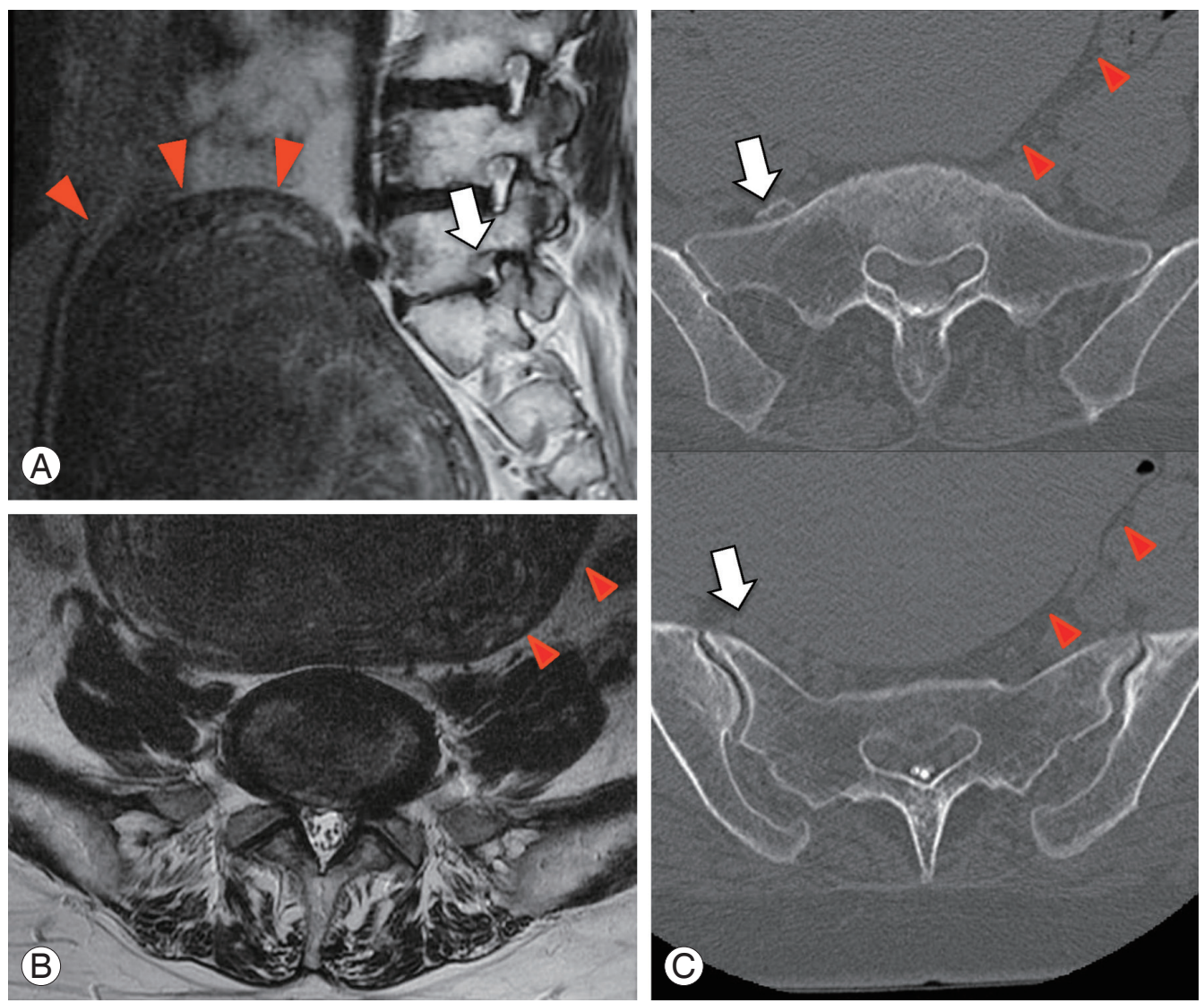

Fig. 1. (A) Lumbar magnetic resonance imaging (MRI) (T2-weighted image, sagittal): massive uterine myoma (red arrowheads) in contact with the anterior surface of the lumbosacral vertebrae. Left L5 foraminal stenosis (white arrow). (B) Lumbar MRI (T2-weighted image, axial): no stenosis in the central region of the spinal canal at L5/S1. (C) Right L5 nerve after contrast CT (axial): right L5 nerve (white arrow) compressed between a massive uterine myoma (red arrowheads) and the sacrum.
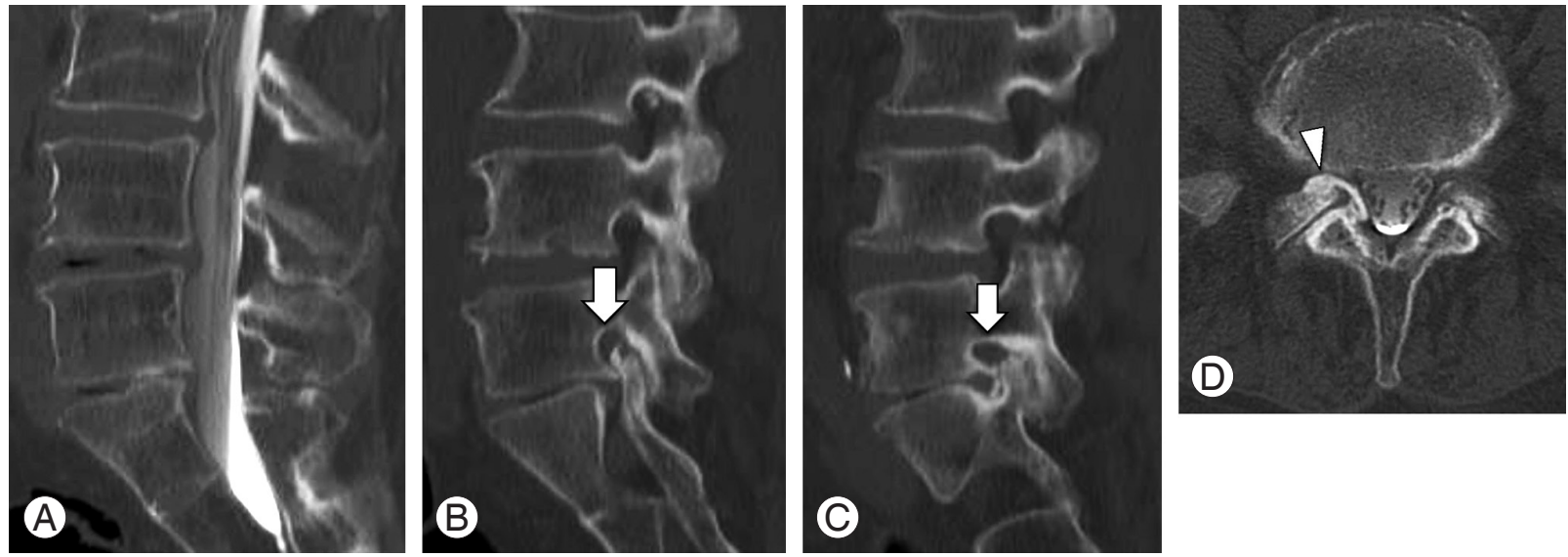

Fig. 2. Post-spinal myelogram computed tomography. (A) Sagittal midline. (B) Parasagittal (right) L5 foraminal stenosis (white arrow). (C) Parasagittal (left) L5 foraminal stenosis (white arrow). (D) L5/S1 axial L5 foraminal stenosis due to right S1 articular process osteophyte (white arrowhead).

$0.021,0.441 \pm 0.017,0.520 \pm 0.020$, respectively [16]. The FA values on both the left and right were lower outside the foramen.
Based on the DTI findings, the patient was diagnosed with bilateral vertebral foraminal stenosis. L5/S1 transforaminal lumbar interbody fusion was done to treat the 
L5 foraminal stenosis. L5/S1 facetectomy on the right was carried out. In addition to an osteophyte, the foramen was extensively compressed by the right S1 superior articular process. The right L5 nerve was decompressed and an intervertebral cage $7 \mathrm{~mm}$ in height was inserted between the L5/S1 intervertebral space, and fixed with a pedicle screw (Fig. 5).
Tibial N
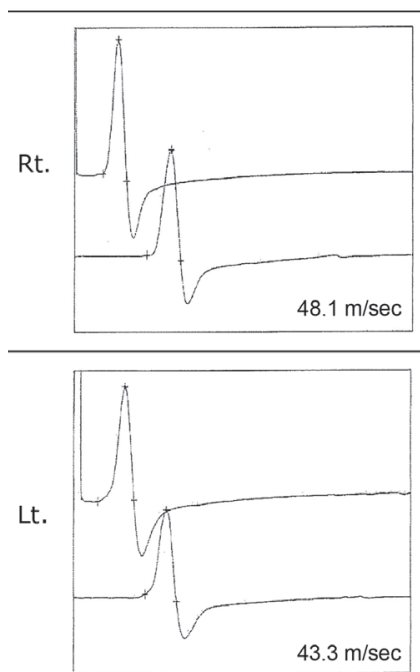

Peroneal N
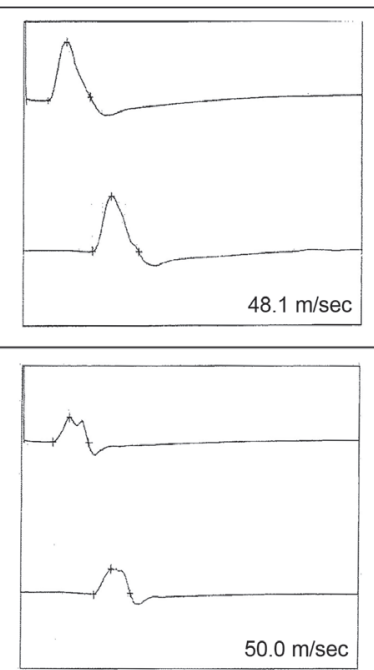

$50.0 \mathrm{~m} / \mathrm{sec}$
Fig. 3. Nerve conduction data.Tibial nerve conduction velocity was $48.1 \mathrm{~m} / \mathrm{sec}$ on the right and $43.3 \mathrm{~m} / \mathrm{sec}$ on the left. Peroneal nerve conduction velocity was $48.1 \mathrm{~m} / \mathrm{sec}$ on the right and $50.0 \mathrm{~m} / \mathrm{sec}$ on the left. There were no abnormalities observed bilaterally.
Immediately after the operation, the pain in both legs disappeared. One year postoperatively, the JOA score was $26 / 29$, VAS for low back pain was 10 , VAS for lower limb pain was 0 , and VAS for lower limb numbness were 0 on both the left and right. Marked improvement was apparent.

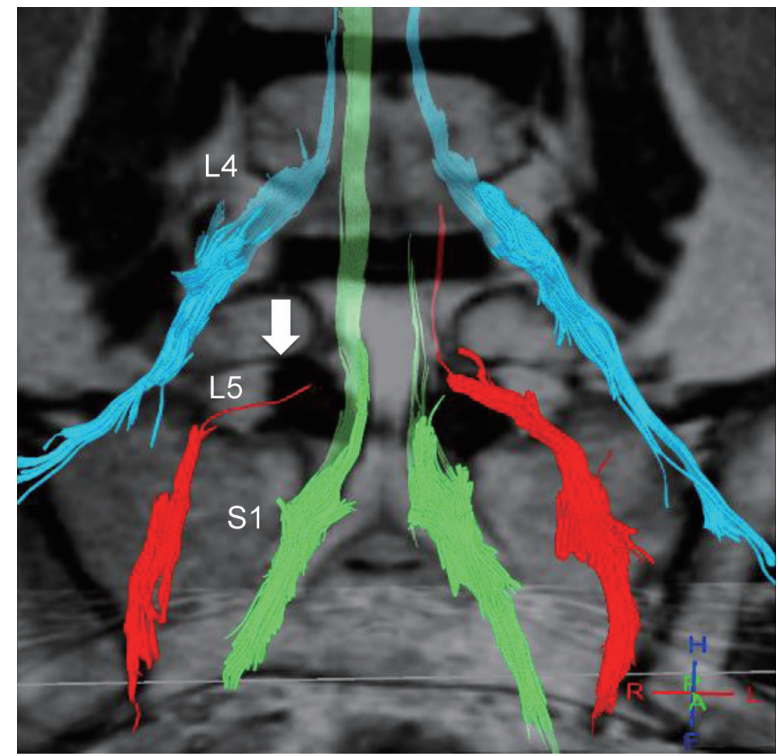

Fig. 4. Fusion image made up of the diffusion tensor tractography of the lumbar nerve $(L 4, L 5, S 1)$ and T2-weighted imaging. Interruption in the right $\mathrm{L} 5$ nerve at the foramen (white arrow).
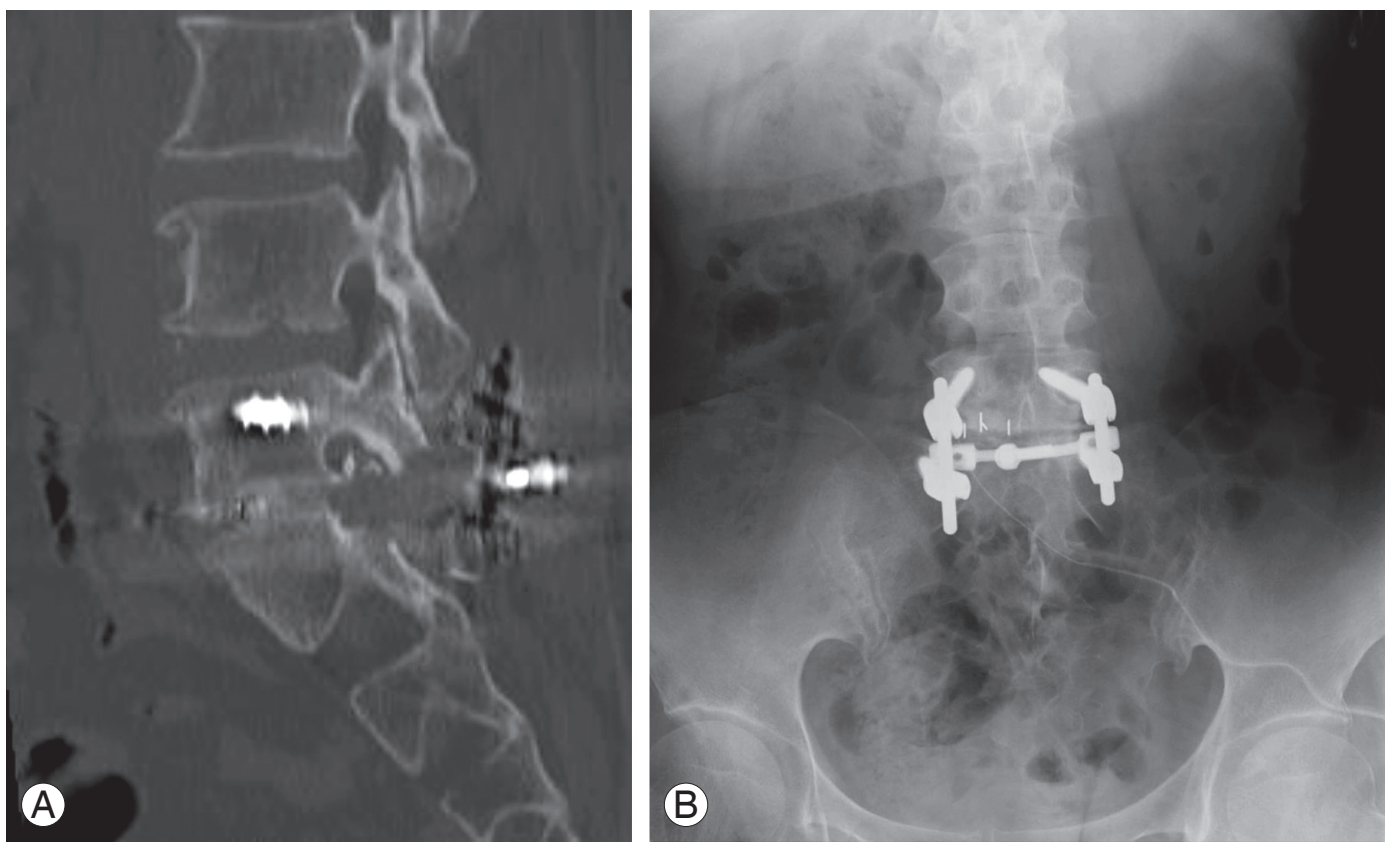

Fig. 5. Plain X-ray after lumbar spine surgery showing (A) sagittal image and (B) anterioposterior image. 


\section{Discussion}

Plain X-ray, CT, and MRI are useful for imaging diagnosis of lumbar foraminal stenosis [17-19], but the rate of false positives is high, and selective nerve root imaging/blocks [20] are used together to reach a comprehensive diagnosis. Recently, the usefulness of three-dimensional (3D)CT, MR myelograpy [3,21], and 3D-MRI [22] have been reported. However, there is no diagnostic method that is useful for determining if the cause of L5 nerve injury is either an L4/5 spinal canal lesion, L5/S1 extraforaminal stenosis, or a double lesion. Electrophysiology used intraoperatively to measure the distal latency of L5 nerve have shown that, compared to a lesion inside the spinal canal, an extraforaminal lesion displayed prolonged distal latency, meaning that it is possible to obtain a diagnosis of foraminal stenosis [23]. However, it is invasive, and there are not yet any noninvasive diagnostic methods. We have reported that tract interruption occurs at the area of lumbar foraminal stenosis when investigated using DTI, and FA values decrease [15].

There are reports of a massive uterine myoma compressing the lumbosacral nerve and causing sciatic neuralgia $[24,25]$. However, in the present case, sciatic neuralgia due to the massive uterine myoma was suspected and a myomectomy was performed, but there was no improvement in the sciatic neuralgia.

No abnormalities of either amplitude or nerve conduction velocity were observed in the tibial or peroneal nerves during electrophysiological studies of the peripheral nerves. Nerve conduction studies are tests that are used to detect nerve conduction disorders peripheral to the knee, but lumbar nerve injuries are localized to the foramen. If Waller degeneration does not extend to the region below the knee, there is the possibility of a falsenegative result [26].

FA values in lumbar foraminal stenosis for extraforaminal lesion were significantly lower when compared to lesions within the spinal canal $(p<0.01)$, and the threshold FA value was 0.42 with a sensitivity of $85.7 \%$ and a false positive rate of $11.1 \%$ with an Odd's ratio of 48 , indicating a high diagnostic precision (manuscript in preparation). Presently, no abnormalities were evident during the nerve conduction tests. Vertebral foraminal stenosis is difficult to diagnose, even when using conventional diagnostic imaging. When DTI was used to investigate the FA values in detail, the FA values were lower and tract interruption was observed in the foramen. Although these FA values were dependent on the site of nerve compression in cases with extraforaminal stenosis, this led to a diagnosis of foraminal stenosis. There have been a few reports of the use of DTI to image lumbar nerve roots [27-30]. Balbi et al. [27] used a Fiber Viewer for nerve injury resulting from herniation of an intervertebral disk, and consecutively measured parameters along the fiber bundle. They reported a precipitous drop in the FA value at the site of compression, which supports our results.

The present case of lumbar foraminal stenosis featured no abnormalities observed during nerve conduction studies, and it was difficult to arrive at a diagnosis using conventional imaging diagnostic methods. DTI was useful in this case. Depending on the site of nerve compression in cases with extraforaminal stenosis, the FA values were lower and tract interruption was observed in the foramen, enabling the diagnosis of foraminal stenosis. We believe that DTI is useful for diagnosing lumbar foraminal stenosis.

\section{Conflict of Interest}

No potential conflict of interest relevant to this article was reported.

\section{References}

1. Jenis LG, An HS. Spine update. Lumbar foraminal stenosis. Spine (Phila Pa 1976) 2000;25:389-94.

2. Macnab I. Negative disc exploration: an analysis of the causes of nerve-root involvement in sixty-eight patients. J Bone Joint Surg Am 1971;53:891-903.

3. Aota Y, Niwa T, Yoshikawa K, Fujiwara A, Asada T, Saito T. Magnetic resonance imaging and magnetic resonance myelography in the presurgical diagnosis of lumbar foraminal stenosis. Spine (Phila Pa 1976) 2007;32:896-903.

4. Basser PJ, Jones DK. Diffusion-tensor MRI: theory, experimental design and data analysis: a technical review. NMR Biomed 2002;15:456-67.

5. Beaulieu C, Allen PS. Determinants of anisotropic water diffusion in nerves. Magn Reson Med 1994;31: 394-400.

6. Beaulieu C, Does MD, Snyder RE, Allen PS. Changes in water diffusion due to Wallerian degeneration in peripheral nerve. Magn Reson Med 1996;36:627-31. 
7. Basser PJ, Pierpaoli C. Microstructural and physiological features of tissues elucidated by quantitativediffusion-tensor MRI. J Magn Reson B 1996;111:20919.

8. Minematsu K, Li L, Fisher M, Sotak CH, Davis MA, Fiandaca MS. Diffusion-weighted magnetic resonance imaging: rapid and quantitative detection of focal brain ischemia. Neurology 1992;42:235-40.

9. Lin X, Tench CR, Morgan PS, Constantinescu CS. Use of combined conventional and quantitative MRI to quantify pathology related to cognitive impairment in multiple sclerosis. J Neurol Neurosurg Psychiatry 2008;79:437-41.

10. Mamata H, Jolesz FA, Maier SE. Apparent diffusion coefficient and fractional anisotropy in spinal cord: age and cervical spondylosis-related changes. J Magn Reson Imaging 2005;22:38-43.

11. Khalil C, Hancart C, Le Thuc V, Chantelot C, Chechin D, Cotten A. Diffusion tensor imaging and tractography of the median nerve in carpal tunnel syndrome: preliminary results. Eur Radiol 2008;18: 2283-91.

12. Lehmann HC, Zhang J, Mori S, Sheikh KA. Diffusion tensor imaging to assess axonal regeneration in peripheral nerves. Exp Neurol 2010;223:238-44.

13. Takagi T, Nakamura M, Yamada M, et al. Visualization of peripheral nerve degeneration and regeneration: monitoring with diffusion tensor tractography. Neuroimage 2009;44:884-92.

14. Mac Donald CL, Dikranian K, Bayly P, Holtzman D, Brody D. Diffusion tensor imaging reliably detects experimental traumatic axonal injury and indicates approximate time of injury. J Neurosci 2007;27: 11869-76.

15. Eguchi Y, Ohtori S, Orita S, et al. Quantitative evaluation and visualization of lumbar foraminal nerve root entrapment by using diffusion tensor imaging: preliminary results. AJNR Am J Neuroradiol 2011;32: 1824-9.

16. Eguchi Y, Ohtori S, Suzuki M, et al. Discrimination between lumbar intraspinal stenosis and foraminal stenosis using diffusion tensor imaging parameters: preliminary results. Asian Spine J. Forthcoming 2016.

17. Hasegawa T, An HS, Haughton VM, Nowicki BH. Lumbar foraminal stenosis: critical heights of the intervertebral discs and foramina. A cryomicrotome study in cadavera. J Bone Joint Surg Am 1995;77:32-8.
18. Kirkaldy-Willis WH, Wedge JH, Yong-Hing K, Tchang S, de Korompay V, Shannon R. Lumbar spinal nerve lateral entrapment. Clin Orthop Relat Res 1982;(169):171-8.

19. Vanderlinden RG. Subarticular entrapment of the dorsal root ganglion as a cause of sciatic pain. Spine (Phila Pa 1976) 1984;9:19-22.

20. Herron LD. Selective nerve root block in patient selection for lumbar surgery: surgical results. J Spinal Disord 1989;2:75-9.

21. Krudy AG. MR myelography using heavily T2weighted fast spin-echo pulse sequences with fat presaturation. AJR Am J Roentgenol 1992;159:1315-20.

22. Yamada $H$, Terada $M$, Iwasaki $H$, et al. Improved accuracy of diagnosis of lumbar intra and/or extraforaminal stenosis by use of three-dimensional MR imaging: comparison with conventional MR imaging. J Orthop Sci 2015;20:287-94.

23. Iwasaki $H$, Yoshida $M$, Yamada $H$, et al. A new electrophysiological method for the diagnosis of extraforaminal stenosis at L5-S1. Asian Spine J 2014;8:145-9.

24. Murphy DR, Bender MI, Green G. Uterine fibroid mimicking lumbar radiculopathy: a case report. Spine (Phila Pa 1976) 2010;35:E1435-7.

25. Murata Y, Takahashi K, Murakami M, Moriya H. An unusual cause of sciatic pain. J Bone Joint Surg Br 2001;83:112-3.

26. Ando M, Tamaki T, Kawakami M, et al. Electrophysiological diagnosis using sensory nerve action potential for the intraforaminal and extraforaminal L5 nerve root entrapment. Eur Spine J 2013;22:833-9.

27. Balbi V, Budzik JF, Duhamel A, Bera-Louville A, Le Thuc V, Cotten A. Tractography of lumbar nerve roots: initial results. Eur Radiol 2011;21:1153-9.

28. van der Jagt PK, Dik P, Froeling M, et al. Architectural configuration and microstructural properties of the sacral plexus: a diffusion tensor MRI and fiber tractography study. Neuroimage 2012;62:1792-9.

29. Budzik JF, Verclytte S, Lefebvre G, Monnet A, Forzy $\mathrm{G}$, Cotten A. Assessment of reduced field of view in diffusion tensor imaging of the lumbar nerve roots at 3 T. Eur Radiol 2013;23:1361-6.

30. Dallaudiere B, Lincot J, Hess A, et al. Clinical relevance of diffusion tensor imaging parameters in lumbar disco-radicular conflict. Diagn Interv Imaging 2014;95:63-8 